Supporting information

\title{
Hydrophilic, Clean Graphene for Cell Culture and Cryo-EM Imaging
}

Jincan Zhang, ${ }^{*}{ }^{\infty}$ Kaicheng Jia, ${ }^{\infty}$ Yongfeng Huang, Yanan Wang, Nan Liu, Yanan Chen, Xiaoting Liu, Xiaojun Liu, Yeshu Zhu, Liming Zhen, Heng Chen, Fushun Liang, Mengqi Zhang, Xiaojie Duan, Hongwei Wang, Li Lin, * Hailin Peng, * and Zhongfan Liu* 


\section{METHODS.}

\section{Growth and transfer of graphene.}

Graphene films were grown on 4 inch-sized single-crystal $\mathrm{Cu}(111) /$ sapphire substrates in the hot-wall chemical vapor deposition (CVD) system, following the previous work reported by our group. ${ }^{1}$ To improve the cleanness of graphene, $\mathrm{CO}_{2}$ was introduced into the CVD chamber after graphene growth at the temperature of $480{ }^{\circ} \mathrm{C}$, which was maintained for 120 min to etch away the amorphous carbon contamination on graphene surface. ${ }^{2}$ Graphene was then transferred onto $\mathrm{SiO}_{2} / \mathrm{Si}$, sapphire and quartz substrates using the optimized poly(methyl methacrylate) (PMMA)-assisted wet transfer method, ${ }^{3}$ while the suspended graphene membrane on the commercial transmission electron microscopy (TEM) grids was fabricated via a polymer-free transfer method. ${ }^{4}$

\section{Characterization.}

The morphology and structure of graphene were characterized using optical microscopy (OM, Olympus BX51), scanning electron microscopy (SEM, FEI Quattro S, acceleration voltage 1-30 kV), TEM (FEI Tecnai F30, acceleration voltage $300 \mathrm{kV}$ and FEI Titan Cubed Themis G2 300, acceleration $300 \mathrm{kV}$ ), atomic force microscopy (AFM, Bruker dimension icon, ScanAsyst mode), white light interferometer (WLI, Nikon, BW-S501) and Raman spectrometer (Horiba, LabRAM HR-800, $532 \mathrm{~nm}$ laser wavelength, $\times 100$ objective). The crystallographic orientation of $\mathrm{Cu}$ substates was measured by electron back-scattered diffraction (EBSD, Zeiss Merlin operated at 20 $\mathrm{kV}$ voltage). The water contact angle (WCA) measurements were performed using a Dataphysics OCA 20 contact-angle system. 


\section{Molecular dynamic (MD) and density functional theory (DFT) simulation.}

$\mathrm{Cu}(111)$-supported monolayer graphene was utilized to simulate the intrinsic water wettability of graphene and the 5-8-5 topological defect was employed as the simplified model of the surface contamination. ${ }^{2}$ The size of graphene was set as $19.921 \mathrm{~nm} \times 20.022 \mathrm{~nm} \times 20.000 \mathrm{~nm}$, containing 15,228 carbon atoms. To elucidate the influence of surface contamination on the water wettability of graphene, 5-8-5 defects were put on the surface of graphene with a uniform distribution, the coverage of which was changed from $0 \%, 5 \%, 10 \%, 20 \%, 40 \%$, to $50 \%$. The L-J parameters for carbon atom were $\sigma_{\mathrm{C}}=0.3689 \mathrm{~nm}$ and $\varepsilon_{\mathrm{C}}=0.5365 \mathrm{~kJ} / \mathrm{mol}$, respectively, consistent with the reported results by Ma et al. ${ }^{5}$ and Werder et $a l .{ }^{6}$ For the MD simulations, a water droplet, which was set as $4 \mathrm{~nm} \times 4 \mathrm{~nm} \times 4 \mathrm{~nm}$ and contained 2,109 water molecules, was placed on each structure with a certain coverage of surface contamination. Specifically, the vertical distance from the water droplet to graphene plane was $0.5 \mathrm{~nm}$, and the MD simulation ran for $6 \mathrm{~ns}$ with the time step of 2 fs to fulfill the equilibrium. After the equilibrium, the average WCA was obtained using the data collected in the final 2 ns. Note that, all the MD simulations were conducted with the Gromacs 4.5.5 package under the temperature of $298 \mathrm{~K}$ and the water model was SPC. ${ }^{7,8}$ The cutoff for van der Waals interactions and electrostatic interactions were both $1.0 \mathrm{~nm}$ and the electrostatic interaction was calculated by using the Particle-mesh Ewald method. ${ }^{9}$

The DFT calculation was performed with the plane-wave pseudopotential code "Vienna ab initio simulation package", ${ }^{10-12}$ employing the two-leg configuration and the Perdew-Burke-Ernzerhof exchange-correlation functional in generalized gradient approximation (GGA). ${ }^{13}$ To describe the electron-nuclear interactions, the projector- 
augmented wave method was used, ${ }^{14}$ with van der Waals interactions included in the simulations by using the D2 approach. ${ }^{15}$ The clean and unclean graphene/ $\mathrm{Cu}(111)$ models were established with Brillouin zone sampled at the $\Gamma$ point. In detail, the clean graphene/ $\mathrm{Cu}(111)$ model was constructed by a $(8 \times 8) \mathrm{Cu}(111)$ surface supercell (containing $192 \mathrm{Cu}$ atoms) and a $(8 \times 8)$ graphene supercell ( containing $182 \mathrm{C}$ atoms), and the unclean graphene/ $\mathrm{Cu}(111)$ model was constructed by adding a 5-8-5 topological defect (containing $52 \mathrm{C}$ atoms) to the top of the graphene/ $\mathrm{Cu}(111)$ model. ${ }^{2}$ Considering the lattice mismatch between the $\mathrm{Cu}(111)$ and graphene, the size of the supercell model utilized in this work was $1.968 \mathrm{~nm} \times 1.968 \mathrm{~nm}\left(\phi=120^{\circ}\right)$. An energy cutoff of $500 \mathrm{eV}$ was employed, with the periodically repeated slabs separated by a vacuum of $3 \mathrm{~nm}$. The structures were relaxed until the maximum force on each atom was less than $0.02 \mathrm{eV}^{-1}$.

\section{Cell culture and data analysis.}

HEK 293 cells were cultured in Dulbecco's modification of Eagle's medium supplied with $10 \%$ fetal bovine serum, $100 \mathrm{U} / \mathrm{mL}$ penicillin, and $100 \mathrm{U} / \mathrm{mL}$ streptomycin under $5 \% \mathrm{CO}_{2}$ condition. The HEK 293 cells were seeded at the unclean graphene, clean graphene and bare quartz substrates and then cultured for $48 \mathrm{~h}$, respectively. To label the cell nuclei with fluorescent probe, the fixed cells were first stained with Hoechst 33342 (sigma B2261, $5 \mu \mathrm{g} / \mathrm{ml}$ ) and then imaged by fluorescence microscopy (X71, Olympus, Japan). Finally, the cell number counting was conducted with the assistance of NIH ImageJ software.

\section{Cryoelectron microscopy (EM) experiment and data analysis.}


A droplet of PNPase solution $(\sim 4 \mu \mathrm{L})$ was pipetted onto the clean graphene and unclean graphene grids, respectively, and then blotted for $2 \mathrm{~s}$ at the force of -2 in Thermo Fisher Scientific Vitrobot, with the humidity of $100 \%$ and the temperature of $12{ }^{\circ} \mathrm{C}$. Afterwards, the graphene grids were plunge-frozen into liquid ethane and kept in liquid nitrogen. Single-particle cryo-EM data sets were collected on an FEI Titan Krios equipped with a Thermo Fisher Scientific Falcon III direct electron detector camera at an accelerating voltage of $300 \mathrm{kV}$ and the TEM images were acquired at a

defocus range of -2.0 to $-3.2 \mu \mathrm{m}$ with a magnified physical pixel size of $0.88 \AA$. Every micrograph was dose-dependently fractionated into 32 frames, at a total accumulated dose of $\sim 50 \mathrm{e}^{-2}$. CTF estimation and defocus values calculation were performed by package CTFFIND ${ }^{16}$ and the results were manually inspected. A total of 244,011 particles were picked, normalized, and iteratively two dimensional (2D) classified by Relion $3.1^{16}$. After discarding particles grouped in poorly defined 2D classes, 15,136 particles were left for further reconstruction and refinement. After postprocessing, we finally got a 6 Å resolution density map estimated by Fourier Shell Correction (FSC) 0.143 cutoff criteria.

\section{SUPPLENMENTARY NOTE}

\section{Section 1. Basic characterization of the single-crystal graphene wafer}

To eliminate the interference originated from the rough substrate, single-crystal $\mathrm{Cu}(111)$ film was firstly fabricated via solid-phase epitaxial process (Figure S1a). As shown in Figure S1b,c, AFM and EBSD characterization confirmed the high flatness and crystallinity of the as-prepared $\mathrm{Cu}(111)$ film, which was beneficial for the growth of single-crystal graphene wafer with decreased density of grain boundaries and 
wrinkles. To explore the influence of the surface contamination on graphene wettability, clean graphene wafer was prepared using $\mathrm{CO}_{2}$ post-treatment technology since $\mathrm{CO}_{2}$ could selectively etch away the amorphous carbon contamination without damaging graphene lattice. ${ }^{2} \mathrm{OM}$ and Raman characterization in Figure S1d-f confirmed the high quality of the clean graphene wafer, as evidenced by the noiselevel D-band intensity in both the Raman spectra and the mapping results of the intensity ratio of $\mathrm{D}$ to $\mathrm{G}$ bands $\left(\mathrm{I}_{\mathrm{D}} / \mathrm{I}_{\mathrm{G}}\right)$.
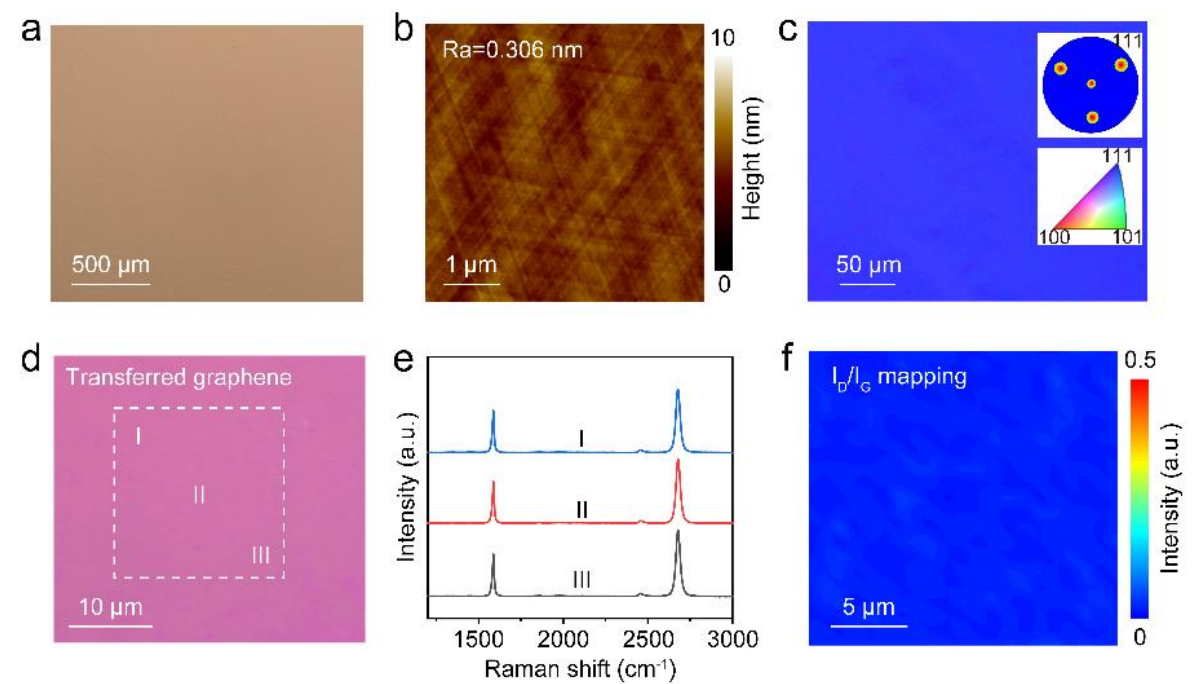

Figure S1. Characterization of the graphene film grown on $\mathrm{Cu}(111)$ substrate. (a) Representative OM image of the single-crystal $\mathrm{Cu}(111)$ film on sapphire wafer. (b) Representative AFM image of the $\mathrm{Cu}(111)$ film. (c) Representative EBSD mapping result of $\mathrm{Cu}(111)$ film. (d) $\mathrm{OM}$ image of the clean graphene wafer transferred onto $\mathrm{SiO}_{2} / \mathrm{Si}$ substrate. (e) Raman spectra of the clean graphene transferred onto a $\mathrm{SiO}_{2} / \mathrm{Si}$ substrate, as marked in (d). (f) $I_{D} / I_{G}$ mapping result of the square region marked in (d).

\section{Section 2. Basic characterization of the suspended graphene film}


To evaluate the intrinsic cleanness of graphene wafer without the interference of polymer residues, the as-synthesized graphene wafer was transferred from $\mathrm{Cu}(111)$ substrate to a commercial TEM grid via a polymer-free transfer method. ${ }^{4}$ The TEM grid used in this work is $3 \mathrm{~mm}$ in diameter, including square-shaped meshes and circular holes with diameter of $2 \mu \mathrm{m}$ (Figure S2a,b). The low-magnification TEM images in Figure S2c,d displayed a uniform contrast indicating the absence of transfer-induced residues. The intensity ratio of the first and second order of the diffraction points in the selected area electron diffraction (SAED) pattern further confirmed the monolayer feature of the suspended graphene film (Figure S2e).

However, amorphous carbon contamination was noticeable with darker contrast in the high-magnification TEM image and its areal proportion can be further calculated by dying the contaminated regions (Figure S2f-g).

a

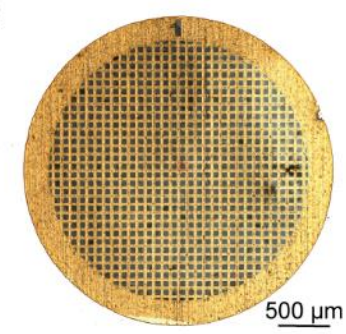

b

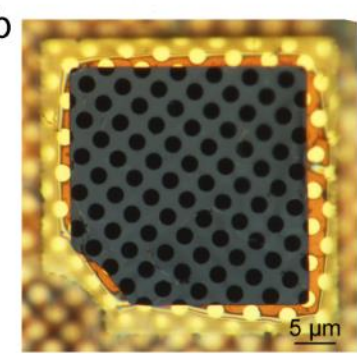

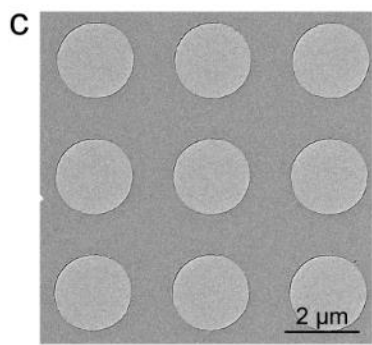
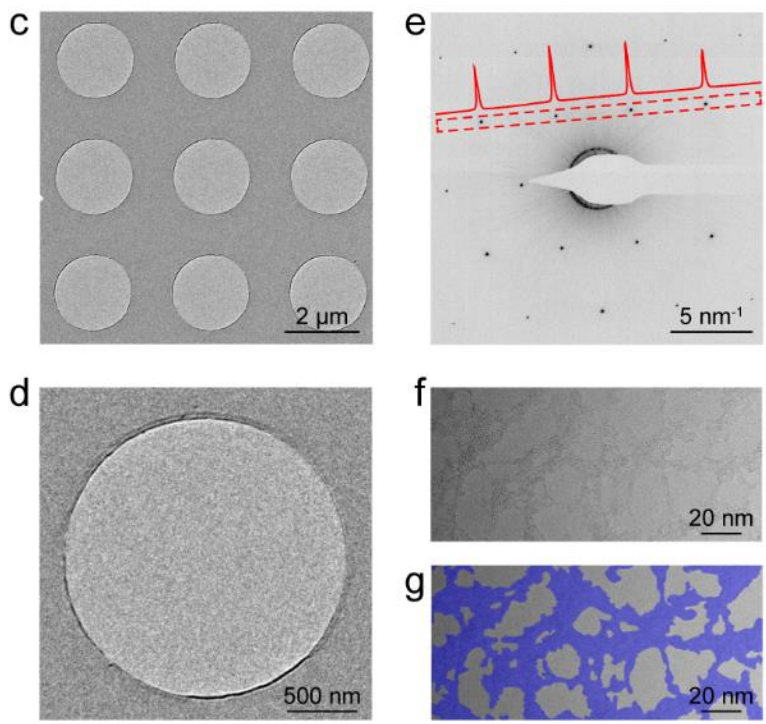
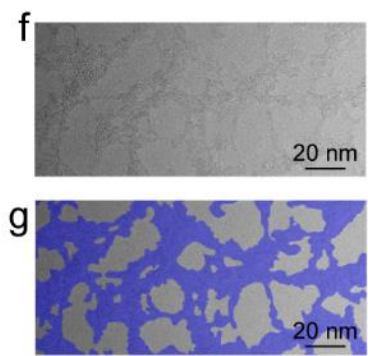

Figure S2. Characterization of the suspended graphene film. (a,b) Low-magnification (a) and high-magnification (b) OM images of the suspended graphene membrane transferred onto a commercial TEM grid. (c,d) Low-magnification TEM images of the suspended graphene film. (e) Representative SAED pattern of the monolayer 
graphene film. (f,g) High-magnification TEM image of the unclean graphene film (f) and corresponding image with the contaminated regions dyed in semi-transparent blue color (g).

\section{Section 3. Time-dependent WCA evolution of the unclean and clean graphene/Cu}

To compare the water wettability of clean and unclean graphene samples, static WCA measurements were conducted by carefully controlling their exposure time in ambient air, in consideration of the impact of airborne hydrocarbon contamination.

Representative WCA measurement results after exposure to air for different time were shown in Figure S3 and S4. Note that, the difference in WCA values of clean and unclean graphene was always larger than $20^{\circ}$ during the measurement, indicating the potential for comparing the graphene cleanness in large-area with the aid of water visualization. Moreover, owing to the nearly stable WCA values after exposure in air for $60 \mathrm{~min}$, uniform distribution of the water droplets on a 4 inch-sized single-crystal graphene/Cu sample was observed, with the variation in WCA values smaller than $5^{\circ}$ (Figure S5), further confirming the potential in large-area fast evaluation of the graphene cleanness in a nondestructive manner.

The wettability of graphene after treatment using $\mathrm{CO}_{2}$ at varied temperature and with different time was measured after taking out of the CVD chamber for $2 \mathrm{~min}$, as displayed in Figure S6. Specifically, after treatment at $480{ }^{\circ} \mathrm{C}$ for $0 \mathrm{~min}, 30 \mathrm{~min}$, and $120 \mathrm{~min}$, the WCA values of graphene were $63^{\circ}, 54^{\circ}$, and $36^{\circ}$, respectively, showing clear dependence on the initial cleanness of graphene on $\mathrm{Cu}$. Meanwhile, after $\mathrm{CO}_{2}$ treatment at $200{ }^{\circ} \mathrm{C}, 300{ }^{\circ} \mathrm{C}, 400{ }^{\circ} \mathrm{C}$ for $120 \mathrm{~min}$, the WCA values of graphene were $63^{\circ}, 62^{\circ}$, and $48^{\circ}$, respectively, further indicating the importance of temperature in altering the graphene wettability. These results indicate that the parameters utilized in 
$\mathrm{CO}_{2}$ treatment would affect the graphene wettability, which would also affect the potential applications of graphene.
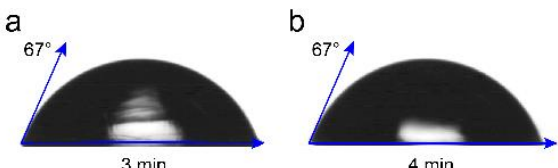

e
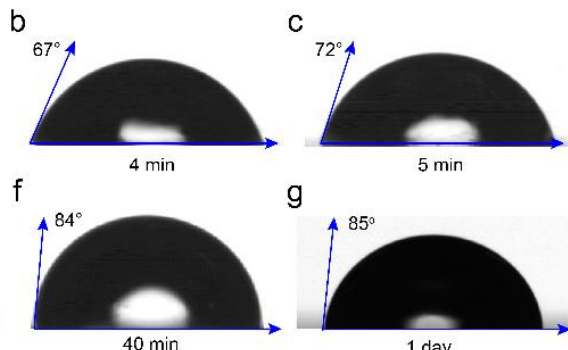

g
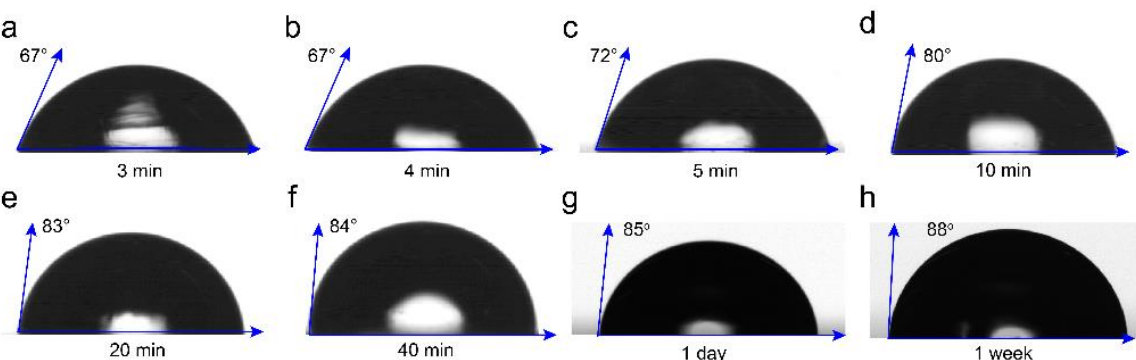

h

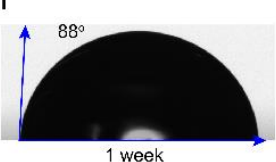

Figure S3. Time evolution of the water wettability of the unclean graphene/Cu. (a-h)

WCA measurement results of the unclean graphene/ $\mathrm{Cu}$ after exposure in air for $3 \mathrm{~min}$ (a), $4 \min (b), 5 \min (c), 10 \min (d), 20 \min (e), 40 \min (f)$, one day (g), and one week (h).
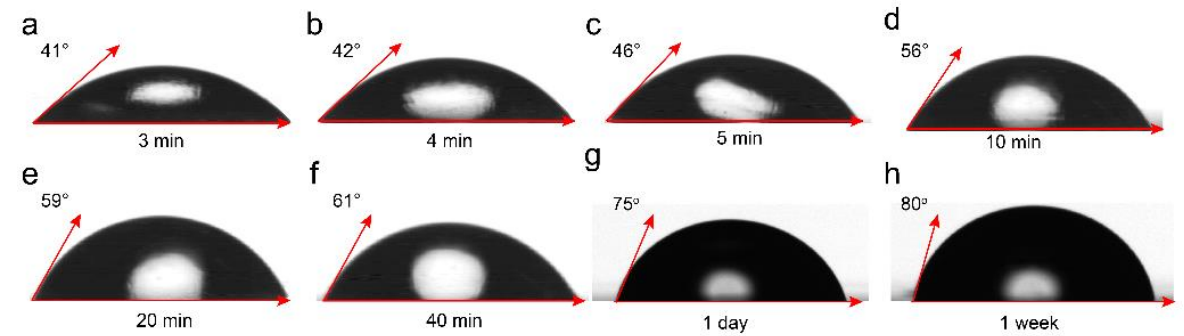

Figure S4. Time evolution of the water wettability of the clean graphene/Cu. (a-h) WCA measurement results of the clean graphene/ $\mathrm{Cu}$ after exposure in air for $3 \mathrm{~min}$ (a), $4 \min (b), 5 \min (c), 10 \min (d), 20 \min (e), 40 \min (f)$, one day (g), and one week (h). 


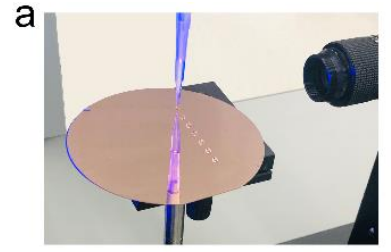

b

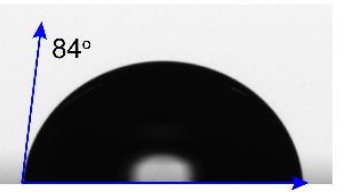

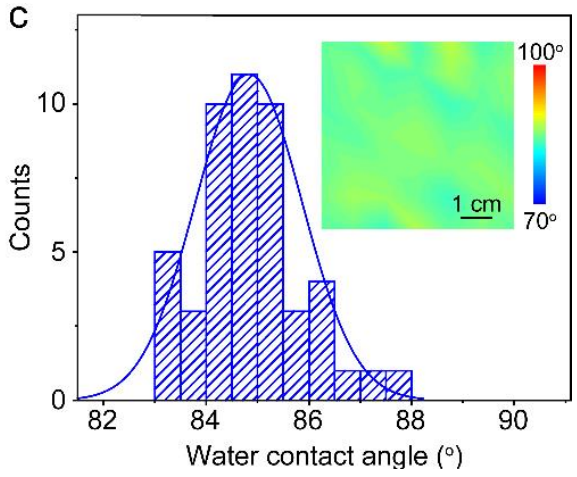

Figure S5. Wettability evaluation of a 4 inch-sized unclean graphene/Cu sample after exposure in air for $60 \mathrm{~min}$. (a) Photograph of the water droplets on 4 inch-sized unclean graphene/Cu surface. (b) Representative WCA measurement result. (c) Histogram statistics of the WCA values on the 4 inch-sized unclean graphene/Cu. Inset: WCA mapping result.

a

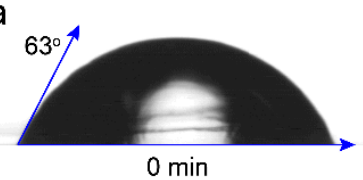

d

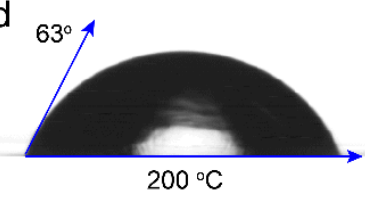

b

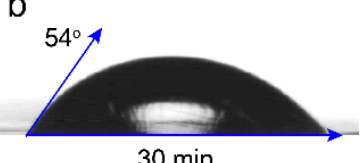

e

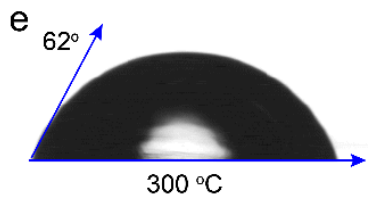

$\mathrm{C}$

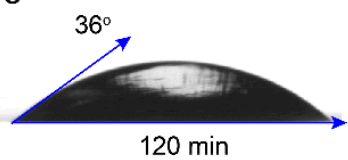

f

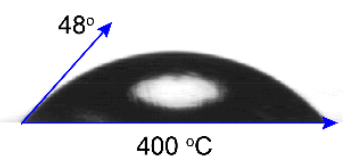

Figure S6. Representative WCA measurement results of graphene after $\mathrm{CO}_{2}$ treatment with different parameters. (a-c) WCA measurement results of graphene after $\mathrm{CO}_{2}$ treatment at $480{ }^{\circ} \mathrm{C}$ for $0 \mathrm{~min}$ (a), $30 \mathrm{~min}$ (b), and $120 \mathrm{~min}$ (c). (d-f) WCA measurement results of graphene after $\mathrm{CO}_{2}$ treatment at $200{ }^{\circ} \mathrm{C}$ (d), $300{ }^{\circ} \mathrm{C}$ (e), and $400{ }^{\circ} \mathrm{C}$ (f) for 120 $\min$.

Section 4. MD simulations of the water wettability of $\mathrm{Cu}$-supported graphene 
MD simulation was performed to investigate the distribution of the water molecules on the $\mathrm{Cu}$-supported graphene. The structures of $\mathrm{Cu}$-supported clean and unclean graphene for the MD simulations are displayed in Figure S7 and S8. In detail, $\mathrm{Cu}(111)$ was utilized as the metal substrate underneath graphene (Figure S7a), on which the $\mathrm{C}_{3 \mathrm{v}}$-symmetry graphene lattice was well-aligned (Figure $\mathrm{S} 7 \mathrm{~b}$ ), consistent with previously reported structures. ${ }^{17,18}$ Meanwhile, the 5-8-5 defect structure was utilized as the simplified model of the amorphous carbon contamination on graphene surface (Figure S7c-d). ${ }^{2,19}$ The structures of graphene with different coverage of amorphous carbon contamination were demonstrated in Figure S8, where the coverage of surface contamination was defined by the areal ratio of the total size of these 5-8-5 defective structures to the whole size of the graphene film. Representative structures of the clean and unclean graphene on $\mathrm{Cu}$ before water deposition was presented in Figure S7, and the apparent dependence of WCA on the surface cleanness of graphene was shown in Figure 2a-c.
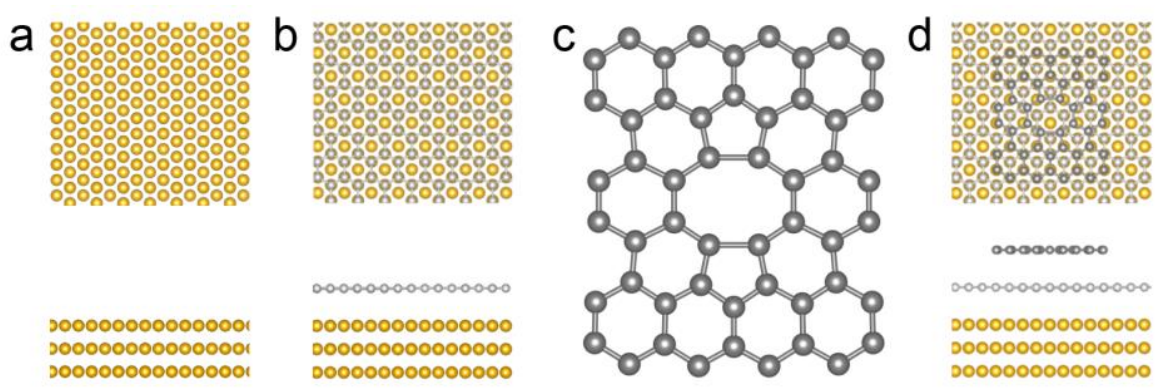

Figure S7. Construction of the Cu-supported clean and unclean graphene structures for the water wettability simulations. (a) Structure of the $\mathrm{Cu}(111)$ substrate. (b) Structure of the $\mathrm{Cu}(111)$-supported clean graphene. (c) Structure of the 5-8-5 defective structure, which is utilized as the simplified model of amorphous carbon contamination. (d) Representative structure of the $\mathrm{Cu}(111)$-supported unclean graphene. Note that $\mathrm{Cu}$ atoms correspond to the yellow spheres, and $\mathrm{C}$ atoms in the 
graphene and contamination are represented by light grey and dark grey spheres, respectively.
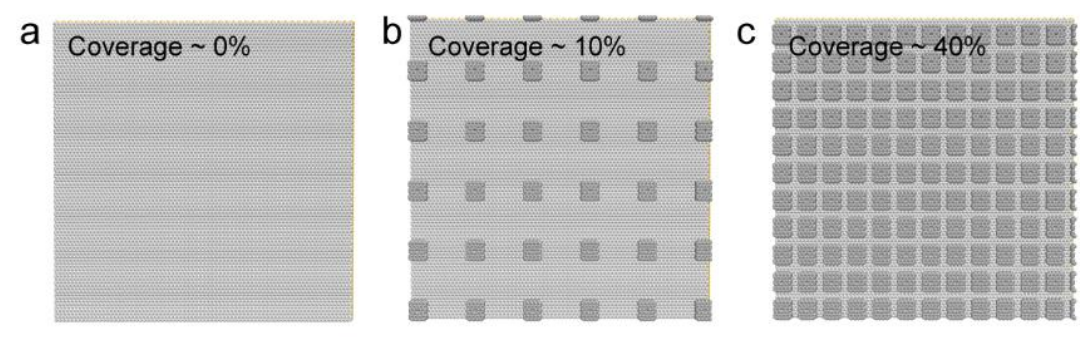

Figure S8. Representative graphene/Cu(111) structures with varied cleanness for the MD simulations. (a-c) Large-area top views of the $\mathrm{Cu}(111)$-supported clean graphene (a) and unclean graphene with the contamination coverage being $10 \%$ (b), and $40 \%$ (c), respectively.

\section{Section 5. DFT calculations of the interaction between water and graphene}

The interaction between the adsorbed water molecules and graphene was investigated by first-principle DFT simulations, using the configurations of two-leg model, where the water molecule stands vertically with two $\mathrm{H}-\mathrm{O}$ bonds pointing toward the carbon atoms in the graphene plane. ${ }^{5}$ We firstly classified the adsorption of water molecules into three conditions according to their projective positions on the graphene/Cu, including 1) atop clean graphene/Cu, 2) atop the contamination on unclean graphene $/ \mathrm{Cu}$, and 3) atop graphene aside the contamination in the unclean graphene $/ \mathrm{Cu}$ system. Stable adsorption sites of water molecules on graphene were then found by geometry optimization, based on which, the adsorption energy was also obtained.

As shown in Figure S9a, the water molecule adsorbed at the center of a carbon hexagon in the graphene lattice was found to be most stable, rather than at the top or bridge adsorption sites, in agreement with previously reported results. ${ }^{5,20}$ 
Subsequently, the most stable adsorption height of the water molecule on the graphene surface was confirmed by finding the minimum adsorption energy along the $z$ axis, that is, along the surface normal direction. Note that, in all the constructed structures, height of the adsorbed water molecule was defined as the vertical distance from its $\mathrm{O}$ atom to the graphene plane. Similarly, the adsorption sites of water molecules on the unclean graphene were also confirmed, as shown in Figure 4d and Figure S9b,c. Moreover, the height of the water molecules was $0.314 \mathrm{~nm}$ in the first and third conditions, and $0.646 \mathrm{~nm}$ in the second condition, with the surface contamination being $0.339 \mathrm{~nm}$ higher than the graphene plane and $0.307 \mathrm{~nm}$ lower than the water molecule.

The adsorption energy of water molecules, which is highly related with the water wettability of graphene, was calculated using the following equation:

$$
E_{\text {ads }}=E_{\text {tot }}\left(\mathrm{H}_{2} \mathrm{O}-\mathrm{G} / \mathrm{Cu}\right)-E_{\mathrm{tot}}(\mathrm{G} / \mathrm{Cu})-E_{\mathrm{tot}}\left(\mathrm{H}_{2} \mathrm{O}\right)
$$

where $E_{\mathrm{tot}}\left(\mathrm{H}_{2} \mathrm{O}-\mathrm{G} / \mathrm{Cu}\right)$ and $E_{\mathrm{tot}}(\mathrm{G} / \mathrm{Cu})$ are the total energies per supercell of the watergraphene/Cu and graphene/Cu systems, respectively. The $E_{\mathrm{tot}}\left(\mathrm{H}_{2} \mathrm{O}\right)$ is the energy of an isolated water molecule. As shown in Figure 2i, the adsorption energy of the water molecules on the unclean graphene were all larger than that on the clean graphene, indicating weaker interactions between the water molecule and the contaminated graphene, and thus more hydrophobic surface of the unclean graphene than its clean counterpart. 

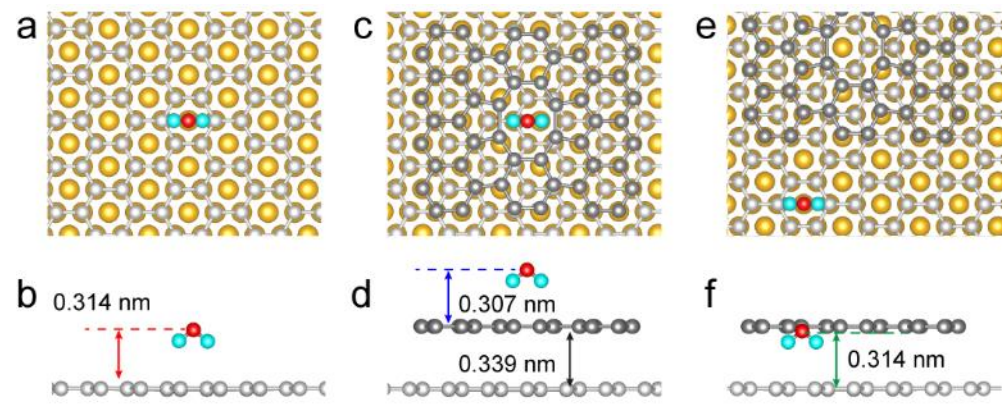

0000000

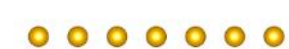

f

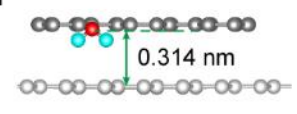

, 0000006

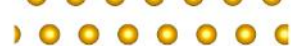

0000000 .

0000000

Figure S9. Two-leg configurations of the water molecules adsorbed on the clean and unclean graphene. (a,b) Top (a) and side (b) views of the water molecule adsorbed on the clean graphene/Cu. (c,d) Top (c) and side (d) views of the water molecule adsorbed on top of the contamination on the unclean graphene/Cu. (e,f) Top (e) and side (f) views of the water molecule adsorbed on top of the graphene surrounded by the contamination on the unclean graphene/ $\mathrm{Cu}$. The $\mathrm{Cu}, \mathrm{O}$, and $\mathrm{H}$ atoms correspond to the yellow, red, and blue spheres, respectively. $\mathrm{C}$ atoms in the graphene and contamination are represented by light grey and dark grey spheres, respectively

\section{Section 6. Application of the clean graphene in cell culture}

To compare the surface roughness of the clean and unclean graphene transferred on quartz substrates, WLI measurement was conducted. As shown in Figure S10a, the clean graphene exhibited a flat surface and negligible contamination was observed. In contrast, there was dense distribution of surface contamination on the unclean graphene, and the particle height reached several nanometers (Figure S10b,c). Meanwhile, WCA measurement and cell culture results of bare quartz substrate were provided in Figure S11, which further indicated the advantages of clean graphene to function as the plate support for cell culture. 

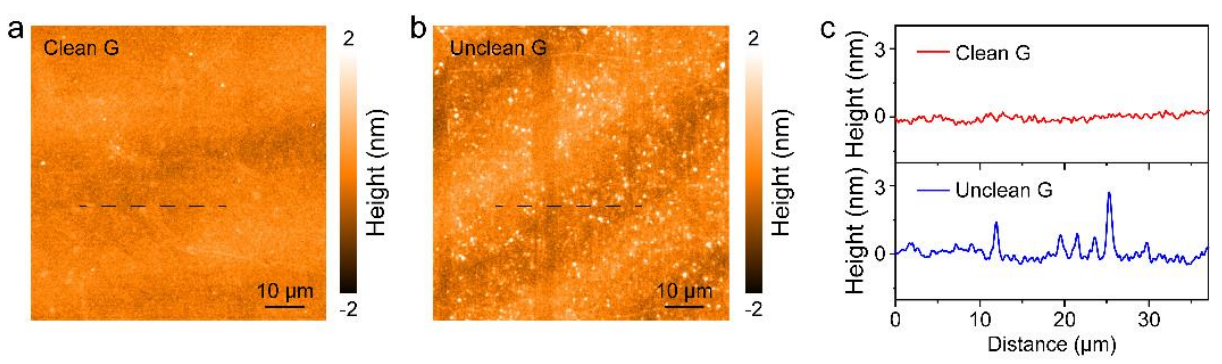

Figure S10. Surface cleanness evaluation of the graphene after transfer with the assistance of PMMA. (a,b) Representative WLI images of clean (a) and unclean (b) graphene after transfer onto the sapphire substrates. (c) Height profiles along the dashed black lines in (a) and (b), corresponding to the clean (red) and unclean (blue) graphene surface, respectively.
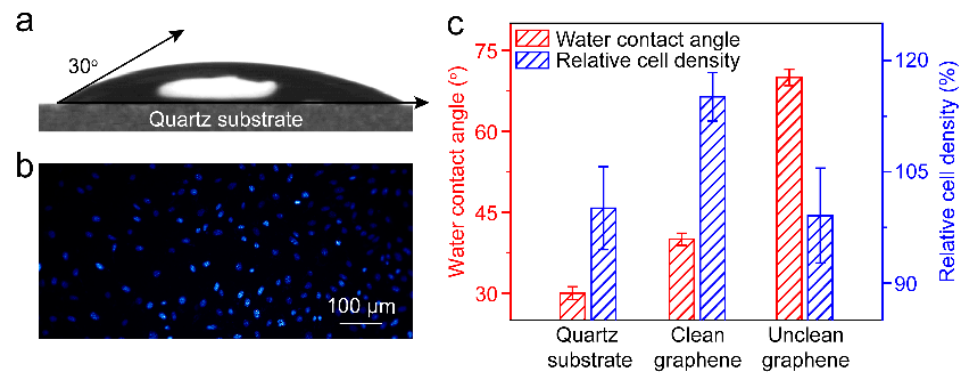

Figure S11. Cell culture using the quartz substrates as the reference plate support. (a) WCA measurement result of the bare quartz substrate. (b) Fluorescence microscope image of cultured cells on the quartz substrate after proliferation for $48 \mathrm{~h}$. (c) Comparison of the WCA values (red) and the density of cultured cells (blue) on quartz, clean graphene/quartz, and unclean graphene/quartz substrates.

\section{Section 7. Application of the clean graphene in cryo-EM imaging}

The suspended graphene before and after water deposition were both characterized using SEM. As shown in Figure S12a, before water deposition, the boundary between the suspended and supported graphene was clear, accompanied by a weak contrast difference between the graphene-covered and graphene breakage regions, while after 
the formation of uniform water membrane on the clean graphene surface, an obvious contrast difference between the regions with and without graphene appeared, while the edges of the suspended graphene became unsharp (Figure S12b). Meanwhile, the original cryo-EM image without dying the PNPase particles distributing on clean suspended graphene was shown in Figure S12c as a supplementary. Moreover, the statistical result of the density of PNPase particles on the suspended clean and unclean graphene surface was given in Figure S12d, furthering indicating the advantages of the clean graphene for cryo-EM imaging. The reconstruction result of the PNPase using clean graphene as the supporting membrane was also shown in meshed style in Figure S13a,c. Meanwhile, the Fourier shell correlation (FSC) curve of the three-dimensional reconstruction result further confirmed a high imaging resolution of $\sim 0.65 \mathrm{~nm}$.
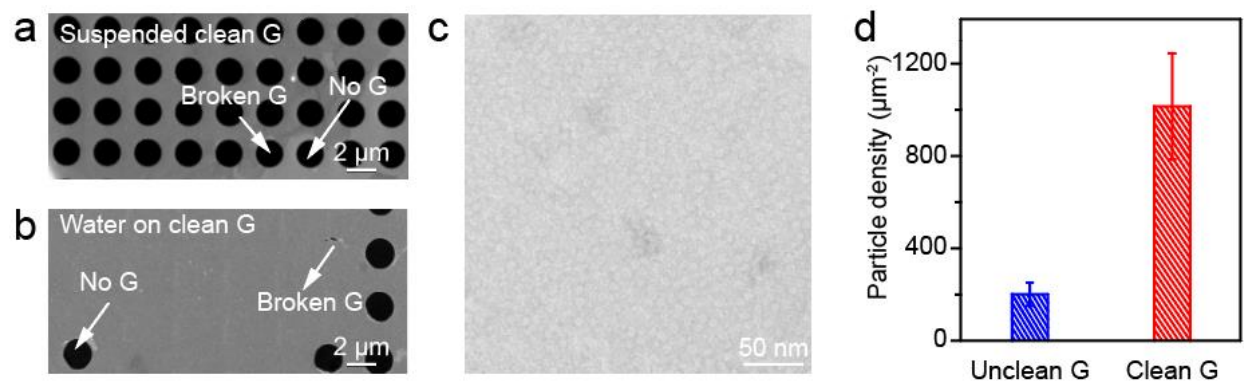

Figure S12. Distribution of water on the surface of clean graphene. (a,b) Lowmagnification SEM images of the suspended clean graphene before (a) and after (b) coverage of the uniform water membrane. (c) Cryo-EM image showing the distribution of PNPase particles in a uniform ice layer supported by the clean graphene. (d) Comparison of the density of PNPase particles on the surface of unclean (blue) and clean (red) graphene. 


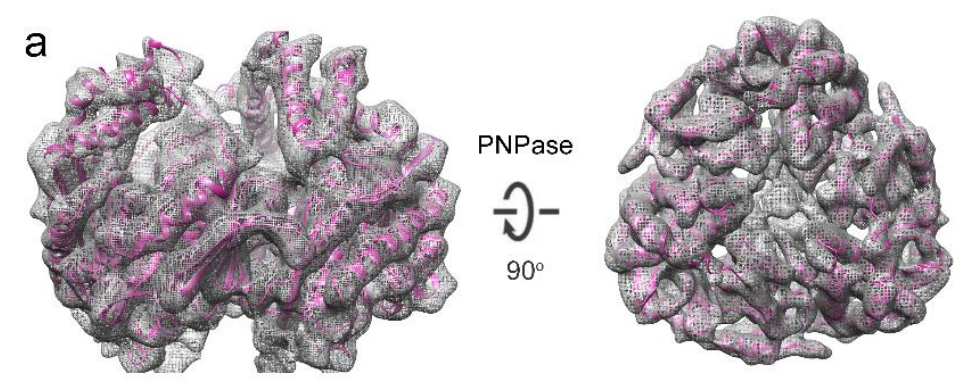

b
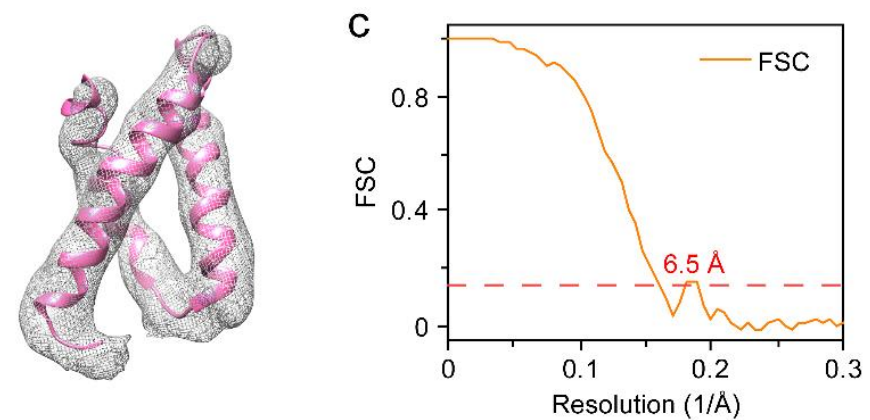

Figure S13. The reconstruction result of PNPase using clean graphene as the supporting membrane. (a) The PNPase density shown in meshed style, with the corresponding model docked. ${ }^{21}$ (b) Selected region of PNPase's map to show the secondary structure. (c) The FSC curve of the three-dimensional reconstruction result. The dotted line indicated FSC $=0.143$.

\section{REFERENCES}

1. Deng, B.; Pang, Z.; Chen, S.; Li, X.; Meng, C.; Li, J.; Liu, M.; Wu, J.; Qi, Y.; Dang, W., Wrinkle-free single-crystal graphene wafer grown on strain-engineered substrates. ACS Nano 2017, 11 (12), 12337-12345.

2. Zhang, J.; Jia, K.; Lin, L.; Zhao, W.; Quang, H. T.; Sun, L.; Li, T.; Li, Z.; Liu, X.; Zheng, L.; Xue, R.; Gao, J.; Luo, Z.; Rummeli, M. H.; Yuan, Q.; Peng, H.; Liu, Z., Large-Area synthesis of superclean graphene via selective etching of amorphous carbon with carbon dioxide. Angew. Chem. Int. Ed. Engl. 2019, 58 (41), 1444614451. 
3. Reina, A.; Son, H.; Jiao, L.; Fan, B.; Dresselhaus, M. S.; Liu, Z.; Kong, J., Transferring and identification of single-and few-layer graphene on arbitrary substrates. J. Phys. Chem. C 2008, 112 (46), 17741-17744.

4. Zhang, J.; Lin, L.; Sun, L.; Huang, Y.; Koh, A. L.; Dang, W.; Yin, J.; Wang, M.; Tan, C.; Li, T.; Tan, Z.; Liu, Z.; Peng, H., Clean Transfer of large graphene single crystals for high-intactness suspended membranes and liquid cells. Adv. Mater. 2017, $29(26), 1700639$.

5. Ma, J.; Michaelides, A.; Alfè, D.; Schimka, L.; Kresse, G.; Wang, E., Adsorption and diffusion of water on graphene from first principles. Phys. Rev. B 2011, 84 (3). 6. Werder, T.; Walther, J. H.; Jaffe, R.; Halicioglu, T.; Koumoutsakos, P., On the water-carbon interaction for use in molecular dynamics simulations of graphite and carbon nanotubes. J. Phys. Chem. B 2003, 107 (6), 1345-1352.

7. Berendsen, H. J.; Postma, J. P.; van Gunsteren, W. F.; Hermans, J., Interaction models for water in relation to protein hydration. Springer, Dordrecht, 1981, 331-342. 8. Hess, B.; Kutzner, C.; Van Der Spoel, D.; Lindahl, E., GROMACS 4: algorithms for highly efficient, load-balanced, and scalable molecular simulation. J. Chem. Theory Comput. 2008, 4 (3), 435-447.

9. Darden, T.; York, D.; Pedersen, L., Particle mesh Ewald: An N· $\log (\mathrm{N})$ method for Ewald sums in large systems. J. Chem. Phys. 1993, 98 (12), 10089-10092.

10. Kresse, G.; Hafner, J., Ab initio molecular dynamics for liquid metals. Phys. Rev. B: Condens. Matter Mater. Phys. 1993, 47 (1), 558-561.

11. Kresse, G.; Hafner, J., Ab-initio molecular-dynamics for open-shell transitionmetals. Phys. Rev. B: Condens. Matter Mater. Phys. 1993, 48 (17), 13115-13118. 
12. Kresse, G.; Hafner, J., Ab initio molecular-dynamics simulation of the liquidmetal-amorphous-semiconductor transition in germanium. Phys. Rev. B: Condens. Matter Mater. Phys. 1994, 49 (20), 14251-14269.

13. Perdew, J. P.; Burke, K.; Ernzerhof, M., Generalized gradient approximation made simple. Phys. Rev. Lett. 1996, 77 (18), 3865-3868.

14. Kresse, G.; Joubert, D., From ultrasoft pseudopotentials to the projector augmented-wave method. Phys. Rev. B: Condens. Matter Mater. Phys. 1999, 59 (3), $1758-1775$.

15. Grimme, S., Semiempirical GGA-type density functional constructed with a longrange dispersion correction. J. Comput. Chem. 2006, 27 (15), 1787-1799.

16. Shi, Z.; Yang, W.-Z.; Liu-Chao, S.; Chak, K.-F.; Yuan, H. S., Crystal structure of Escherichia coli PNPase: Central channel residues are involved in processive RNA degradation. RNA 2008, 14 (11), 2361-2371.

17. Li, B. W.; Luo, D.; Zhu, L.; Zhang, X.; Jin, S.; Huang, M.; Ding, F.; Ruoff, R. S., Orientation-dependent strain relaxation and chemical functionalization of graphene on a $\mathrm{Cu}$ (111) foil. Adv. Mater. 2018, 30 (10), 1706504.

18. Xu, X.; Yi, D.; Wang, Z.; Yu, J.; Zhang, Z.; Qiao, R.; Sun, Z.; Hu, Z.; Gao, P.;

Peng, H., Greatly enhanced anticorrosion of $\mathrm{Cu}$ by commensurate graphene coating. Adv. Mater. 2018, 30 (6), 1702944.

19. Banhart, F.; Kotakoski, J.; Krasheninnikov, A. V., Structural defects in graphene. ACS Nano 2011, 5 (1), 26-41.

20. Li, X.; Feng, J.; Wang, E.; Meng, S.; Klimeš, J.; Michaelides, A., Influence of water on the electronic structure of metal-supported graphene: Insights from van der Waals density functional theory. Phys. Rev. B 2012, 85 (8), 085425. 
21. Shi, Z.; Yang, W.-Z.; Liu-Chao, S.; Chak, K.-F.; Yuan, H. S., Crystal structure of Escherichia coli PNPase: Central channel residues are involved in processive RNA degradation. RNA 2008, 14 (11), 2361-2371. 